\title{
Design and Operation of Very Slow-Speed Generators for a Bristol Cylinder Sea Wave Generating Device
}

\author{
Sze Song Ngu \\ Universiti Malaysia Sarawak \\ Sarawak, 94300, Malaysia \\ ssngu@feng.unimas.my
}

\author{
David G. Dorrell \\ Unviersity of Technology Sydney \\ Sydney, NSW 2007, Australia \\ David.dorrell@uts.edu.au
}

\author{
C. Cossar \\ University of Glasgow \\ Glasgow, G12 8LT, UK \\ c.cossar@gla.ac.uk
}

\begin{abstract}
This paper describes the operation of a direct-drive brushless generator for a Bristol cylinder ocean wave device. This is a very low speed device so the pole number and diameter is very large. While the machine may be large the pole pitch and axial length is low. The application is described and simulated using analytical and finite element analysis techniques. A 248 pole design with surface rotor magnets is developed with both surface and slotted windings. An analysis of the control is put forward.
\end{abstract}

\section{INTRODUCTION}

Recently, a number of small companies have tried to develop and commercialize a range of varying wave energy devices, and promote the devices as a non-polluting source of energy. The research in this field is now receiving increasing amounts of funding from various governments and related organizations.

The functions of a sea wave energy device are to harness the energy of sea waves and convert the energy into useful forms of energy for domestic or industrial use. It is therefore known as wave energy converter. There are several significant reviews of wave energy devices which describe and discuss the various forms of device developed which attempt to harness sea wave energy. Basically, wave devices can be categorized into several types of device; these categories are:

1. The oscillating water column (OWC).

2. The point absorber.

3. The flap or surge device.

4. The attenuator or contouring device

5. Overtopping devices.

6. Other types that are unique and do not fall into any category above.

The OWC has a semi-submerged structure forming an air chamber with a top outlet though which reciprocating airflow flows through it; this drives the bi-directional turbine. As the incidents wave surface rise inside the chamber, the air will be compressed and go through the top outlet. Some examples of OWCs and component bidirectional turbines are the Limpet and the Breakwater Turbine developed by Wavegen [1][2], the Denniss-Auld
Turbine [3], the Ocean Energy Buoy [4] and the SeWave OWC [5]. Point absorbers refer to a buoy and floats at or near to the wave surface. Wave energy from all directions can be absorbed by the vertical movement of the buoy as the waves pass. Examples of point absorber wave devices are the PowerBuoy [6], the CETO [7] and the Linear Generator [8]. Surge wave devices harness energy from the horizontal movement of the water particles in waves. They are normally situated in shallower water and close to shore and the circular movement of the water becomes elongated into horizontal ellipses. Examples for this form of device are the Oyster [9] and the WaveRoller [10]. Attenuator/Contouring devices are elongated floating devices that are parallel to the wave direction. When incident wave propagates along the device, movement within the device is generated which produces energy. Examples are the Pelamis [11], the Wave Star [12] and the Anaconda [13]. Overtopping devices rely on using a funnel or barrage arrangement on the device to elevate part of the incident waves above the mean sea level to fill a raised reservoir. The seawater returns to the sea via a low-head turbine. Examples of this device are the Wave Dragon [14] and the Multiple Stage Overtopping Device [15]. Some of the wave energy devices need gears and hydraulic systems to generate electricity, while some are direct drive wave energy devices [16]. The wave devices each have their own advantages and disadvantages when compared to the others.

This paper will address the control of a Bristol Cylinder device. In the next section the application conditions are discussed then the Bristol Cylinder is described in more detail. This is followed by a design outline for the generators and then the control will be addressed.

\section{BRISTOL CYLINDER OPERATION}

The Bristol Cylinder wave device that is studied here does not come under any of the categories discussed above. It is a semi-submerged device that is marginally buoyant which rotates synchronously with the incident wave, given an appropriate speed control. The wave power absorption characteristic of a submerged cylinder was studied in [17] and [18]; Fig. 1 shows a basic arrangement. A 